\title{
Anabases
}

ANABASES Traditions et réceptions de l'Antiquité

13 | 2011

Varia

\section{Daniel et Ashpenaz : sur quelques lectures contemporaines de Daniel 1, 7 et 9}

Régis Courtray

\section{OpenEdition}

Journals

Édition électronique

URL : http://journals.openedition.org/anabases/1810

DOI : 10.4000/anabases.1810

ISSN : 2256-9421

Éditeur

E.R.A.S.M.E.

Édition imprimée

Date de publication : 1 mars 2011

Pagination : 55-78

ISSN : 1774-4296

\section{Référence électronique}

Régis Courtray, «Daniel et Ashpenaz : sur quelques lectures contemporaines de Daniel 1, 7 et 9 »,

Anabases [En ligne], 13 | 2011, mis en ligne le 01 mars 2014, consulté le 21 octobre 2019. URL : http:// journals.openedition.org/anabases/1810 ; DOI : 10.4000/anabases.1810

Ce document a été généré automatiquement le 21 octobre 2019

(c) Anabases 


\title{
Daniel et Ashpenaz : sur quelques lectures contemporaines de Daniel 1, 7 et 9
}

\author{
Régis Courtray
}

\section{Daniel et Ashpenaz, couple homosexuel?}

1 Au cours de ces dernières années, les interprétations de la Bible se sont multipliées, dans des domaines où parfois on ne l'attendait pas. C'est ainsi que, devant des littéralistes brandissant l'Écriture comme garante de la morale chrétienne, certains se sont à leur tour emparés de textes bibliques pour prouver que la Bible approuve ce que ces derniers condamnent. Depuis les années 1970, certaines figures bibliques sont ainsi devenues, contre toute attente, des "icônes gay »: on pense bien sûr au "couple " David et Jonathan ${ }^{1}$ ou à leur pendant féminin, Ruth et Noémie, mais moins connu est le troisième : Daniel et Ashpenaz. Il faut dire que celui-ci n'est cité en exemple que depuis une dizaine d'années, et même moins dans le domaine francophone. Deux études récentes nous fourniront les principaux arguments mis en avant ${ }^{2}$.

Le premier chapitre du livre de Daniel raconte comment Daniel et trois jeunes Hébreux - membres de l'aristocratie judéenne - ont été arrachés au royaume de Juda et déportés à la cour de Babylone par le roi Nabuchodonosor. Placés sous la surveillance d'Ashpenaz, chef des eunuques du roi, ils doivent être instruits par ce dernier dans la langue et les lettres des Chaldéens durant trois années avant d'entrer au service du roi. Or, par souci de respecter les prescriptions alimentaires juives, Daniel supplie Ashpenaz de lui épargner la souillure de la nourriture ${ }^{3}$ et de lui donner des légumes à manger et de l'eau à boire. Le livre dit alors littéralement ${ }^{4}$ (Dn 1, 9) : «Et Dieu donna Daniel en

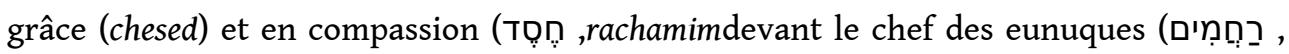

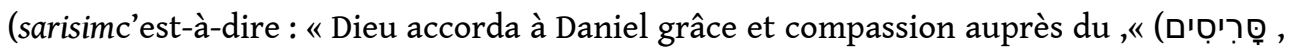
chef des eunuques. » C'est autour de ce verset que s'est bâtie l'interprétation du passage. 
C'est d'abord la traduction des deux mots hébreux chesed et rachamim qui a retenu l'attention. Si le premier ne pose pas de difficulté et signifie " grâce, faveur ", le second est plus ambigu et pourrait être traduit soit par "grâce, pitié, compassion », soit par " amour physique " (" physical love») 5 . L'argumentation alors avancée est que, le texte hébreu ne pouvant dire deux fois la même chose, le sens du mot est nécessairement "amour physique ${ }^{6}$ ». Naturellement, ce sens inacceptable a été gommé par les traducteurs qui ont préféré utiliser des termes plus neutres. De fait, la plupart des traductions françaises ont adopté des expressions comme "grâce et faveur" (тов, Pléiade), "grâce et miséricorde » (Bible de Jérusalem), "grâce et pitié » (Osty) ; seul Chouraqui traduit au plus près de l'étymologie : « chérissement et matrices ${ }^{7}$; dans le domaine anglophone, la King James Version propose : Now God had brought Daniel into favor and tender love with the prince of the eunuchs, proche en cela du sens originel supposé.

Il y a d'autre part les questions soulevées par la notion d'eunuques. Certains voient en Ashpenaz seul un eunuque, d'autres pensent que Daniel et ses compagnons avaient également été faits eunuques. Or, dans la littérature prohomosexuelle nord-américaine, l'eunuque en est venu à désigner, de manière générale, tout homme qui, pour diverses raisons, ne se marie pas et n'a pas d'enfants ${ }^{8}$; par extension, les eunuques pourraient donc correspondre aux " gays ${ }^{9}$ » voire aux transsexuels ${ }^{10}$. Mais, qu'Ashpenaz seul ait été eunuque ou que Daniel l'ait également été, comment concevoir une relation sexuelle? Les uns affirment que les eunuques n'étaient pas nécessairement castrés, mais c'était ainsi qu'on appelait, dans le Moyen-Orient antique, des hommes sexuellement attirés par d'autres hommes ${ }^{11}$; pour d'autres, lorsque des hommes sont castrés après la puberté, ils conservent leurs pulsions sexuelles ${ }^{12}$.

De ces deux arguments, on tire la conclusion qu'il a existé une relation homosexuelle entre Daniel et le chef des eunuques du palais de Nabuchodonosor; "cette aventure amoureuse pourrait expliquer en partie la rapide ascension de Daniel à la cour », ajoute Daniel Helminiak.

6 Telle est donc la thèse que certaines lectures contemporaines soutiennent sur ce début du livre de Daniel. Dans la mesure où le passage se trouve désormais cité sans un examen plus approfondi, il nous semble que la question mérite d'être réexaminée plus à fond. Pour ce faire, nous reprendrons chacun des éléments évoqués en le considérant des points de vue philologique, biblique, historique et exégétique; nous recourrons également aux lectures anciennes de ce même passage qui donneront une profondeur de vue à notre analyse.

\section{La « compassion » d'Ashpenaz pour Daniel}

7 Revenons tout d'abord sur le terme rachamim,Il s'agit d'un mot figé au pluriel (בַחְמִים) dérivé de rechem: qui signifie : « matrice, sein, utérus, entrailles »; il a pour sens (רֶֶֶ) " amour, compassion, grâce, miséricorde, pitié, bonté ». Si l'on analyse l'ensemble de ses occurrences dans l'Ancien Testament ${ }^{13}$, on constate que le mot, s'il peut parfois signifier les entrailles des hommes comme siège de leurs sentiments (1 R 3, 26 ; Pr 12, 10), décrit plus fréquemment la bonté, la compassion de Dieu pour son peuple ou pour l'homme (Dt 13, $18 ; 2$ S 24, $14 ; 1$ Ch 21, 13 ; Ne 9, 19.27.28.31; Ps 25,$6 ; 40,12 ; 51,3 ; 69$, $17 ; 77,10 ; 79,8 ; 103,4 ; 119,77.156 ; 145,9$; Is 54,$7 ; 63,7.15$; Jr 16, 5 ; Lm 3, 22 ; Dn 9, 9.18 ; Os 2,21 ; Za 1, 16). Il est donc l'expression même de l'amour maternel du Créateur pour sa créature. Par suite, il peut qualifier la compassion d'un homme pour un autre, 
mais toujours dans un contexte semblable: Dieu inspire (ou on demande à Dieu d'inspirer) de la compassion aux nations étrangères en faveur de son peuple déporté ou éprouvé (Gn 43, $14.30 ; 1 \mathrm{R} \mathrm{8,} 50$; 2 Ch 30, 9 ; Ne 1, 11 ; Ps 106, 46 ; Jr 42, 12 ; Jdt 10, $8^{14}$ ); par extension, il est utilisé pour demander aux hommes de faire preuve de compassion les uns pour les autres $(\mathrm{Za} 7,9)$; à l'inverse, des textes peuvent souligner que telle nation n'a pas pris en pitié le peuple de Dieu (Is 47,6 ; Am 1,11). Tel est bien le contexte du passage qui présente Daniel comme déporté à la cour de Nabuchodonosor: Dieu vient inspirer à Asphenaz de la compassion à son égard ; celui-ci va devenir une figure bienveillante, rendant sensible au jeune garçon la présence rassurante de Dieu auprès de lui. Il faut encore noter que l'association des mots chesed et rachamim n'est pas un fait isolé ; dans plusieurs autres passages de la Bible, les deux mots se trouvent dans un contexte proche (Ps 25,$6 ; 40,12 ; 51,3 ; 69,17 ; 77,10 ; 103,4 ; 106,45-46 ; 145,8-9$; Is 63,$7 ; \mathrm{Jr} 16,5 ; \mathrm{Lm} 3,22 ;$ Os 2, $21 ; \mathrm{Za} \mathrm{7,9)}$, pour décrire la compassion de Dieu. Il s'agit donc d'une expression bien attestée.

Le débat sur les termes chesed et rachamim n'est pas nouveau. On constate en fait que les anciens avaient tenté de déterminer le sens de chacun des deux mots. Ainsi, dans le Commentaire sur Daniel qu'il rédigea au milieu du XII siècle, André de Saint-Victor traduisait le premier mot par gratia («reconnaissance») et le second par misericordia (" pitié ») : «Il y a "reconnaissance", écrit-il, - au sens où l'on dit : un tel ou un tel a manifesté de la reconnaissance - quand on garde le souvenir des amitiés et des devoirs d'un autre et la volonté de payer un autre de retour. Dans ce passage, elle est à interpréter comme un sentiment gratuit d'affection, une bienveillance sincère et une sainte gratitude, par laquelle le Seigneur a inspiré de la reconnaissance pour Daniel à l'esprit du chef des eunuques. Quant à la "pitié", les stoïciens la définissent comme "un chagrin né du malheur d'un autre, souffrant injustement - car personne n'est ému par le supplice d'un parricide ou d'un traître ${ }^{15 "}$. Le chagrin est, selon eux, "l'opinion actuelle sur un malheur présent à propos duquel il semble juste que l'âme s'abatte et se resserre ${ }^{16 "}$. Ainsi, de manière divine, lui fut inspiré qu'il semble juste à son âme de s'abattre et de se resserrer en raison du malheur de Daniel, autre (i.e. étranger) à son peuple, souffrant injustement. Car il était vraiment évident, même pour un homme sans intelligence, que lui et ses trois compagnons souffraient du malheur de leur captivité présente, non à cause de leurs péchés, mais à cause de ceux d'autres hommes. Car leur âge juvénile n'aurait jamais pu commettre quelque mal que ce soit au point de devoir être fait prisonnier ${ }^{17}$. "

9 Il convient enfin d'insister sur le verset lui-même qui dit clairement non pas qu'Ashpenaz prit en grâce et en compassion Daniel, mais que « Dieu accorda à Daniel grâce et compassion auprès du chef des eunuques ». Les exégètes anciens avaient bien noté le rôle essentiel de Dieu dans la naissance du sentiment de compassion chez le chef des eunuques. Pour Jérôme ${ }^{18}$, par exemple, la faveur dont Daniel bénéficie auprès d'Ashpenaz n'est rien d'autre que la récompense qu'il s'est acquise par ses vertus ; c'est parce qu'il s'était proposé dans son cœur de ne pas se souiller du repas du roi que Dieu, dans sa générosité, lui a fait trouver grâce et miséricorde auprès du chef des eunuques. L'exégète généralise alors son propos : «Nous apprenons par là que, si la nécessité des choses fait que des saints sont aimés par des infidèles, c'est le fait de la miséricorde de Dieu, et non le fait de la bonté d'hommes pervertis. » Au travers de cette phrase, Jérôme applique donc les termes « infidèle » et «perverti » à Ashpenaz. Bien des siècles plus tard, Calvin ${ }^{19}$ affirme quant à lui: «Daniel avoit trouvé grace devant le 
gouverneur, d'autant que Dieu avoit flechi le cœur de cest homme là (qui autrement n'estoit gueres doux ne gratieux) à douceur \& humanité. [...] Il faut noter la façon de parler dont il use ici, à sçavoir que Dieu l'avoit mis en grace et miserations devant ce gouverneur là. Il pouvoit dire à la façon commune qu'il estoit en grace et credit : mais il attribue cela a une grace singuliere de Dieu, qu'il a senti par experience un homme barbare luy estre si humain \& favorable. » Dans ces deux commentaires, la grâce divine est opposée à la malignité d'Ashpenaz, et c'est précisément au travers de cet homme «barbare » et mauvais qu'elle peut s'exprimer dans toute sa latitude.

\section{Ashpenaz était-il eunuque?}

La notion d'eunuque doit à présent être précisée. Le mot hébreu sarisa plusieurs (סָרִ (סָר) sens: il peut désigner l'eunuque au sens d'un homme impuissant par nature (saris hamma, " eunuque par nature») ou par suite d'une castration (saris adam, " eunuque par la main de l'homme »); mais il signifie aussi l'officier, le dignitaire de la cour ; le mot est en effet un emprunt à l'akkadien sha reshi, "celui de la tête ", c'est-à-dire " celui qui est attaché à la personne royale». Le rab sha reshi («eunuque en chef») correspondait, dans l'Empire assyrien, à la fonction de commandant de la cohorte permanente au service du roi $^{20}$. «Il n'est venu à signifier "eunuque" que du fait que l'on confiait souvent cette charge à un eunuque de peur que le majordome n'abusât de la confiance que son maître lui faisait ${ }^{21}$. » Ainsi, il n'est pas toujours facile de déterminer, dans les textes bibliques, si le mot saris doit être traduit d'une manière ou d'une autre. Un article récent a tenté d'éclairer la question ${ }^{22}$, distinguant les passages où il est question d'eunuques au sens propre de ceux où il s'agit de fonctionnaires royaux, d'officiers du palais. Parmi ces derniers se trouve l'« eunuque » Potiphar qui était marié $(G n 39,7)^{23}$ et avait donc peu de chance d'être un véritable eunuque; Potiphar est par ailleurs à deux reprises qualifié de « commandant des gardes » de Pharaon (Gn 37, 36 ; $39,1)^{24}$. Or, cet article range précisément notre passage dans les cas difficiles à déterminer, notant à la fois que le texte se réfère à des officiers de cour - dont fait partie Ashpenaz - ayant en garde les jeunes exilés, mais qu'il devait y avoir de nombreux eunuques à la cour de Babylone, et pas seulement pour garder les femmes ; les auteurs notent d'ailleurs que, dans ce premier chapitre de Daniel, Ashpenaz est nommé archieunouchos ("chef des eunuques») par la Septante et praepositus eunuchorum («préposé des eunuques») par la Vulgate: le terme saris peut ainsi indifféremment désigner un « eunuque » ou un « officier ».

11 La double interprétation du mot saris n'est pas chose nouvelle : on la rencontre dès le judaïsme ancien, et les deux sens sont également appliqués à Ashpenaz : il est soit "préposé sur tous les eunuques", soit « chef des serviteurs» ou «chef militaire ». Au $\mathrm{xvI}^{\mathrm{e}}$ siècle, dans les leçons qu'il a données sur le livre de Daniel, Calvin comprend le mot hébreu au sens figuré : «Les Hebrieux appellent bien en leur langue Sarisim, Eunuques : mais ce nom s'estend a tous gouverneurs. Car Putiphar est ainsi nommé, \& toutefois il estoit marié. Ce nom aussi se trouve souvent en l'Escriture pour gouverneurs de Province : mais a cause que tels gouverneurs estoient choisis d'entre les enfans nobles, il y a apparence, qu'il n'ont esté apellez Eunuques, non pas qu'ils fussent chastrez : mais d'autant que les Eunuques estoient les mignons des Roys d'Orient, de là est advenu comme j'ai desja dit, que les enfans lesquels le Roy nourrissoit comme une semence des principaux de son Royaume, pour puis apres les ordonner gouverneurs en diverses 
provinces, estoient ordinairement appelez de ce nom ${ }^{25}$. » Et voici comment il conçoit la charge d'Ashpenaz : «Car le gouverneur avoit sous soy plusieurs serviteurs et officiers, comme nous sçavons que cela est ordinaire aux grans gouverneurs. Il est vraysemblable que la charge de ce gouverneur là estoit telle qu'est au jourd'huy celle du grand Escuyer, qu'on appelle en France ${ }^{26}$.»

Faudrait-il alors comprendre que le mot "eunuque » désignerait ici des personnes homosexuelles ? C'est ce que semble sous-entendre Daniel Helminiak : «Il existe [...] une forte présomption que les serviteurs de la cour - ou eunuques comme on les appelait dans le Moyen-Orient antique - étaient le plus souvent des hommes dont l'attirance sexuelle était exclusivement dirigée vers des hommes ${ }^{27}$.» Encore faut-il prouver cette idée, avancée ici sans le soutien de sources historiques. Certains appuient leur argumentation sur des exemples de relations homosexuelles entre des personnages historiques et des eunuques : Néron et l'eunuque Sporus (Suétone, Néron XXVIII) ; Darius III et ses eunuques, puis Alexandre le Grand et l'eunuque Bagoas de Darius (PlutARQue, Vie d'Alexandre 67, 8; Quinte-Curce VI, 5, 22-23); Artaxerxès et l'eunuque Téridate (Élien, Histoires variées XII, 1). Mais ces exemples ponctuels ne suffisent pas à généraliser le cas des eunuques. On a encore mis en avant que de nombreux hijra de l'Inde moderne s'offrent comme prostitués pour les hommes; d'autre part, le Kamasutra affirme que tous les eunuques, qu'ils soient efféminés ou plus masculins, sont homosexuels à des degrés plus ou moins grands (II, XI ${ }^{28}$.

L'idée que les eunuques étaient homosexuels pourrait encore faire référence à ceux que les spécialistes de la Mésopotamie ${ }^{29}$ appellent les «professionnels de l'homosexualité passive ", prostitués, cinèdes et invertis regroupés en trois catégories : les assinnu, les kulu'u et les kurgarrû ; ces personnages jouaient un rôle dans la vie liturgique, où ils se travestissaient, jouaient de la musique, chantaient et dansaient, dans des cérémonies en l'honneur d'Ishtar, déesse de l'amour. Or, certains documents porteraient à croire qu'ils étaient eunuques, castrés ou déformés physiquement - mais rien ne prouve qu'ils l'étaient tous. Plus que l'émasculation réelle, c'est toutefois leur comportement efféminé qui semble les caractériser. Mais, si la prostitution en tant qu'institution était considérée comme une prérogative de la haute civilisation, les prostitués, en tant qu'individus, étaient tenus à l'écart et méprisés : on considérait qu'ils avaient dévié de leur destin et de leur norme ; leur comportement passif les assimilait à des femelles et non à des mâles, certains portant même des noms de femmes. Il semble ainsi évident que ces hommes prostitués ne sauraient non plus en aucune manière être assimilés aux eunuques, fonctionnaires royaux, dont fait certainement partie Ashpenaz.

Ce rapide examen des possibilités semble pouvoir établir deux certitudes : le mot saris est ici à prendre plutôt au sens d'une fonction de cour et le texte biblique ne permet en rien de soutenir qu'Ashpenaz était homosexuel.

\section{Daniel était-il eunuque?}

Mais si Ashpenaz n'était pas véritablement eunuque, peut-on affirmer que les jeunes Hébreux avaient été faits eunuques? Jean Steinmann tranche ainsi la question: «Daniel et ses compagnons ne sont pas nécessairement eunuques. [...] Mais les jeunes gens restent célibataires. On ne parle jamais de leurs femmes ni de leurs enfants, ce qui fait contraste avec les renseignements qui nous sont restés au sujet d'anciens prophètes 
comme Osée, Isaïe et Ézéchiel. Il semble que le célibat de Jérémie ait fait école. Daniel et ses compagnons tendent à vivre comme des moines ${ }^{30}$.»

Cependant, l'idée selon laquelle Daniel et ses compagnons étaient eunuques fut très répandue dans le judaïsme ancien ${ }^{31}$. Cette affirmation comporte néanmoins de très nombreuses variantes. Sanhédrin 93b, citant Isaïe $39,7^{32}$, donne deux opinions : soit le mot « eunuques » est à comprendre littéralement (Rab), soit les jeunes Hébreux ont été appelés "eunuques", parce qu'à leur époque, l'idolâtrie avait été complètement retranchée (R. Hanina). Selon cette dernière opinion, Daniel et ses compagnons sont les eunuques dont parle Isaïe 56, 4, qui observent les sabbats de Dieu : en récompense leur sera donné dans la maison de Dieu un monument qui vaut plus que des fils et des filles, un mémorial éternel qui ne sera pas coupé; c'est là, selon le Talmud, une allusion au livre de Daniel, appelé d'après le nom du prophète. Dans le Talmud de Jérusalem ${ }^{33}$, il est précisé, à propos de Daniel 3, 25 que les trois compagnons de Daniel auraient retrouvé leur intégrité physique dans la fournaise de feu (le lien de leur castration a été dénoué). Dans les Pirqé de Rabbi Éliézer (LII, 7), le rapprochement de Daniel 1, 3-7 et Isaïe 39, 7 et 56, 4-5 permet de conclure que Daniel, Ananias, Azarias et Misaël avaient été faits eunuques à la cour de Nabuchodonosor et qu'ils n'ont pas eu d'enfants. Rashi, quant à lui, rapporte, dans son commentaire d'Ézéchiel 14, 3 que Daniel n'a laissé ni fils ni filles. En revanche, d'après Sanhédrin 93a, les trois compagnons de Daniel se sont par la suite mariés et ont eu des enfants (R. Johanan). Dans le Midrash Megillah ${ }^{34}$, à propos d'Esther $4,5^{35}$, il est rapporté qu'accusés devant le roi Nabuchodonosor de mener une vie de débauche et menacés d'être exécutés, Daniel et ses compagnons se seraient eux-mêmes châtrés, démontrant que les charges portées contre eux étaient sans fondement. Flavius Josèphe, dans ses Antiquités juives ${ }^{36}$, affirme que, parmi les enfants juifs déportés à Babylone - et dont font partie Daniel, Ananias, Azarias et Misaël -, certains ont été faits eunuques ; même s'il ne dit explicitement que les quatre jeunes gens sont devenus eunuques, on peut néanmoins supposer que tel était bien le cas. Seul Abraham ibn Ezra n'admet pas que Daniel ait été eunuque; il tire argument du verset: "Des jeunes garçons en qui il n'y eût aucun défaut "; de fait, cette insistance sur leur intégrité physique serait un argument pour affirmer que Daniel et ses compagnons n'ont pas pu être faits eunuques ; un autre argument serait que la sagesse acquise par Daniel et ses compagnons aurait été amoindrie par la castration ${ }^{37}$.

Dans le christianisme ancien, on trouve cette même tradition, explicitement attribuée aux Hébreux. Ainsi, si l'on considère d'abord les écrits des Pères grecs, Origène, dans son Commentaire sur Mathieu 15, 5, affirme que Daniel et ses compagnons ont été faits eunuques à Babylone, s'appuyant sur Isaïe 39,7 et 56,3-5. On trouve cette même opinion dans ses Fragments sur le premier livre des Règnes ${ }^{38}$. Commentant Ézéchiel 14, 16 ${ }^{39}$, l'exégète précise que, si Daniel a bien eu des enfants selon un engendrement spirituel, il n'a pas eu d'enfants selon la chair; la raison en est qu'il a été confié au chef des eunuques - ce qui revient à dire qu'il était lui-même eunuque ${ }^{40}$. De même, dans ses Homélies sur Ézéchiel, il mentionne que Daniel fut fait eunuque à Babylone, selon Isaïe 39, 7 et Ézéchiel 14, 16 ; pourtant, par ses paroles prophétiques et divines, il a engendré de nombreux enfants ${ }^{41}$. Outre Origène, on trouve également cette idée, moins développée, dans le Commentaire sur Daniel de Théodoret de Cyr, qui se contente de rapprocher Daniel 1, 3-4 et Isaïe 39, 7, sans en tirer de conclusion ${ }^{42}$, ou dans les Vies des prophètes d'Épiphane de Salamine ${ }^{43}$; pour ce dernier, cependant, Daniel était à ce point chaste que les Juifs ont cru qu'il était eunuque. 

n'est pas pour surprendre: l'exégète latin est disciple à la fois des Hébreux et d'Origène, dont il traduit en latin, en 381, les homélies sur Ézéchiel. Dans son traité Contre Jovinien, le moine affirme que les Hébreux pensent que Daniel et les trois enfants ont été eunuques ${ }^{44}$. Il appuie également cette opinion sur un rapprochement entre Isaïe 39, 7 et Daniel 1, 3-4 ; le fait que les deux textes parlent d'enfants d'origine royale suffit à prouver qu'ils parlent bien des mêmes enfants; or, puisqu'Isaïe affirme que ces enfants royaux seront faits eunuques, alors Daniel, Ananias, Azarias et Misaël ont bien été faits eunuques. Demeure une question: Daniel a-t-il pu avoir des enfants avant d'être fait eunuque ? De fait, on pourrait objecter qu'en Ézéchiel 14, 14-20, il est dit que Noé, Daniel et Job ne peuvent libérer leurs enfants sur une terre pécheresse. Cet argument ne tient pas aux yeux de Jérôme : il ne s'agit là que d'une hypothèse, et il faut donc comprendre : si ces trois hommes avaient eu des enfants à l'époque d'Ézéchiel, ils n'auraient pas pu les libérer de la désolation du pays. La preuve qu'il s'agit bien d'une hypothèse est que Noé et Job ont vécu plusieurs siècles avant l'époque d'Ézéchiel; quant à Daniel, s'il a bien vécu en même temps qu'Ézéchiel - puisque tous deux ont été conduits en captivité à l'époque du roi Joachim ${ }^{45}$-, il était toutefois un enfant quand il fut déporté ; c'est ce que mentionne clairement le texte biblique, qui précise également qu'au bout de trois ans, il fut introduit auprès du roi: puisqu'il est dit que la déportation eut lieu la troisième année du règne de Joachim, alors Daniel entra au service de Nabuchodonosor la sixième année de son règne - soit au moment où Ézéchiel mentionne son nom ${ }^{46}$. Daniel était donc encore un enfant au moment où il fut déporté, et le prophète ne mentionne donc ses fils que par hypothèse. Preuve est ainsi faite que Daniel a bien été fait eunuque avant même qu'il ait pu avoir des enfants. La matière de l'argumentation est très clairement empruntée à Origène, mais le traitement en reste original. se contente cependant de rappeler que les Hébreux prennent appui sur Daniel 1, 3 et Isaïe 39,7 pour affirmer que Daniel et ses compagnons ont été faits eunuques; il relève toutefois une objection possible dans la suite du verset $4:$ « Des enfants en qui il n'y eût aucun défaut ${ }^{47}$. » Plus loin, à propos de Daniel 6, 5, Jérôme cherche à comprendre quelle est l'accusation que les chefs et les satrapes cherchent à trouver « contre Daniel du côté du roi »; les Hébreux, explique-t-il, ont compris que ce " côté du roi » désigne soit la reine, soit des concubines ou des maitresses du roi : on accuserait donc Daniel d'avoir voulu abuser de la reine ou des femmes entourant le roi. «"Mais ils ne purent trouver, disent-ils, aucun grief ni soupçon", parce qu'il était eunuque, et ils ne pouvaient le convaincre dans une affaire de stupre ${ }^{48}$. " Certes, Jérôme qualifie cette explication de «fable» et lui oppose une interprétation simple (Daniel n'avait rien fait de mal); néanmoins, on retrouve ici le thème de Daniel eunuque, attribué aux Hébreux ${ }^{49}$. Évidemment, Jérôme rappelle encore cette opinion des Hébreux en commentant Isaïe 39, 7, dans son Commentaire sur Isaie ${ }^{50}$.

20 En passant les siècles, on retrouve encore ce débat, au xvIII ${ }^{\mathrm{e}}$ siècle, dans le commentaire d'Augustin Calmet sur le livre de Daniel ; le célèbre exégète, pourtant, ne retient pas la thèse : «On donne communément le nom d'eunuques, aux officiers du palais des Rois d'Orient, parce que pour l'ordinaire, ils estoient véritablement eunuques. Les Juifs prétendent que Daniel, \& ses trois compagnons furent faits eunuques par l'ordre de Nabuchodonosor, en exécution d'une prédiction d'Isaïe qui portoit, que les Rois de 
Babylone prendroient des descendans d'Ezéchias, \& qu'ils les feroient eunuques pour servir dans leur palais (Is 39, 7). Mais cette raison n'est pas concluante, puisque dans cette Prophétie, comme dans le passage, que nous expliquons, le nom d'eunuques peut simplement marquer celui qui a un emploi à la cour ${ }^{51}$. »

Il semble donc difficile de déterminer si Daniel et ses trois compagnons ont bien été faits eunuques : la tradition irait dans ce sens, mais cette tradition semble remise en cause dès l'époque moderne et les exégètes contemporains se contentent prudemment de dire qu'ils "ne sont pas nécessairement eunuques». Quoi qu'il en soit, de deux choses l'une : soit ils ont été véritablement été faits eunuques, et toute la tradition y voit le signe de leur continence; soit ils n'ont pas été faits eunuques, et l'argument avancé ne tient plus.

\section{Analyse contextuelle du passage}

Si nous reprenons à présent le texte biblique du point de vue de son contexte, on remarque que le personnage d'Asphenaz apparaît comme très secondaire, matérialisant - on l'a vu - la présence bienveillante de Dieu auprès du jeune prophète. Il est présenté comme assez inconsistant, et on l'a parfois même décrit comme " un peu falot n'osant pas prendre de risques. [...] Finalement, c'est grâce au "garde", fonctionnaire subalterne, que tout s'arrangera ${ }^{52} »$. De fait, dans le texte biblique, il est évident qu'Ashpenaz n'a finalement pas accédé à la demande de Daniel et de ses compagnons de ne pas prendre part à la nourriture et au vin du roi. Si Dieu accorde à Daniel de trouver grâce et miséricorde auprès du chef des eunuques, ce dernier redoute de devenir coupable aux yeux du roi si celui-ci constate que les jeunes Hébreux sont en moins bonne santé que les autres enfants dont il a la charge ; il faut donc comprendre qu'il refuse la requête de Daniel. Or, c'est finalement auprès du garde ${ }^{53}$ que le chef des eunuques leur avait préposé que les jeunes gens vont obtenir gain de cause (Dn 1, 10-16).

23 Les auteurs anciens ont très souvent souligné cet aspect du récit. Chez Théodoret de $\mathrm{Cyr}^{54}$, Ashpenaz est décrit comme un homme qui ne connaît rien à la grâce spirituelle, mais considère toute chose d'un point de vue humain : pour lui, des corps ne peuvent être nourris et ne peuvent grandir qu'avec des aliments riches; la crainte du chef des eunuques est donc fondée, et il redoute les pires châtiments de la part du roi. L'homme est donc mû par la peur, et, constatant cela, Daniel transfère sa prière à son gardien Amalsar. Pour Jean Chrysostome ${ }^{55}$, il n'y avait d'ailleurs aucune raison que le chef des eunuques respectât Daniel: il n'était à ses yeux qu'un enfant méprisable et un prisonnier de guerre; et quand bien même il aurait été respectable, le danger ne lui permettait pas de le voir comme tel : aussitôt après la mention de sa pitié pour Daniel, le texte parle d'ailleurs de sa crainte du roi. Ainsi, puisque rien n'était possible au niveau humain, c'est la grâce céleste qui est venue tout régler. Selon Éphrem le Syrien $^{56}$, puisqu'Ashpenaz ne s'était pas laissé persuader par Daniel, c'est Dieu luimême qui a disposé Amelsad à accéder à sa demande. Dans les Chroniques de Sulpice Sévère $e^{57}$, il n'est pas dit clairement que c'est le garde qui accède à la demande de Daniel ; mais, aussitôt après qu'" Asphane " a exprimé ses craintes et que Daniel a promis qu'il aurait un visage plus beau en mangeant des légumes plutôt que les mets du roi, on lit : «On ajouta foi à ces paroles ${ }^{58}$ »; le garde n'étant jamais mentionné, on peut supposer que c'est «Asphane» lui-même qui accepte de mettre à l'épreuve les 
enfants ${ }^{59}$. En revanche, Flavius Josèphe ${ }^{60}$ considère explicitement qu' « Aschanès ${ }^{61}$ " était prêt à accéder à leur désir, tout en craignant que le roi, s'apercevant de leur maigreur, ne lui fasse subir un châtiment. L'intermédiaire qu'est le garde disparait ici, et les jeunes Hébreux, voyant la bonne disposition de l'eunuque, ont raison de ses scrupules et le persuadent de les nourrir selon le régime alimentaire demandé; constatant l'heureux résultat, "Aschanès " perd alors toute crainte et prend dès lors pour lui tous les aliments que le roi envoie chaque jour aux jeunes gens. Plus tard, au temps des Réformes, Calvin note que le gouverneur «Aspenaz » a refusé ce que Daniel lui demandait : son «humanité » à l'égard du jeune Hébreu n'a donc pas consisté à accéder à sa requête, mais seulement à ne pas l'accuser devant le roi. «Car combien qu'il refuse la requete qu'on luy avoit faite, toutefois il s'excuse gracieusement et avec douceur, comme s'il disoit qu'il accorderoit cela volontiers, s'il ne craignoit d'iriter le Roy contre luy ${ }^{62}$. Or, il aurait pu faire mourir Daniel et ses compagnons sur-le-champ, en les dénonçant au roi. Toutefois, ne pouvant obtenir du gouverneur ce qu'il désirait, Daniel s'est tourné vers son serviteur "Melzar ». Cette idée se rencontre encore au XVIII ${ }^{e}$ siècle sous la plume d'Augustin Calmet: «Le Chef des eunuques avoit refusé à Daniel ce qu'il lui avoit demandé mais Malazar fut plus traitable ${ }^{63}$. " L'ensemble de ces commentaires montre clairement qu'Ashpenaz a rarement été vu comme un adjuvant de Daniel ; tout au plus n'est-il pas hostile au projet des enfants, mais c'est sa crainte de Nabuchodonosor qui le dissuade de leur accorder ce qu'ils lui demandent. C'est donc plutôt le garde, un subalterne, qui aide les jeunes Hébreux.

On pourrait d'autre part trouver un double d'Ashpenaz dans le maître, puis le geôlier auprès desquels le patriarche Joseph a trouvé grâce (Gn 39, $4 ; 21)$ : faudrait-il alors supposer une liaison entre le patriarche et ces deux hommes? Bien plutôt, c'est la femme de Putiphar qui a poursuivi Joseph de ses désirs tandis que, lui, est resté chaste. En fait, le parallèle entre Daniel et Joseph n'est pas fortuit, et il faut certainement voir en Daniel un nouveau Joseph : comme lui, il s'est trouvé en nation étrangère ; comme lui, il a reçu un nouveau nom; comme lui, il a attiré la bienveillance de ceux qui en avaient la garde; comme lui, il a possédé le don d'interpréter les songes. Tous ces parallèles - déjà remarqués par les Pères de l'Église ${ }^{64}$ - sont une volonté de l'auteur biblique de rapprocher les deux personnages. D'autres parallèles pourraient encore être proposés : Esther, par exemple, trouva faveur (chez) et grâce (chesed) auprès du roi Assuérus (Est 2,17) ; Daniel est aussi un nouveau Moïse : il est comme lui instruit dans une culture étrangère.

L'intérêt de cet épisode est encore de montrer que l'observation de la Loi procure à Daniel et à ses compagnons une meilleure santé qu'aux autres enfants et une sagesse supérieure à celle des magiciens et devins du royaume de Nabuchodonosor; les six premiers chapitres de Daniel vont tous dans ce même sens d'une exaltation de la figure du prophète, refusant de se soumettre à d'autres lois qu'à celles de son Dieu. Supposer une relation homosexuelle entre le juif Daniel et un homme étranger, donc païen, serait donc faire fi du sens le plus évident du texte. D'autre part, Daniel n'est pas présenté ici comme un personnage historique dont la vie affective aurait son intérêt, mais comme un instrument de la volonté divine. Effectivement, Daniel et ses compagnons ont parfois été vus comme des « héros sans âme quasiment réduits au rôle de marionnettes. [...] Si les personnages de Daniel ne sont guère vivants, c'est qu'aux yeux de l'auteur, ils sont sans grande importance. Leur personnalité s'évanouit dans la thèse qu'ils sont chargés d'illustrer. Cette thèse [...] consiste à affirmer la nécessité d'une purification pour accéder à la connaissance des mystères ${ }^{65} »$. De fait, le livre s'intéresse moins à la 
personne de Daniel qu'à ses visions prophétiques et à l'issue théologique de la justice de Dieu dans le monde.

Le danger des lectures contemporaines que nous venons d'exposer réside dans le fait qu'elles veulent tirer le texte biblique vers un sens idéologique, anachronique et réducteur. Les travaux récents sur l'homosexualité dans la Bible rappellent que la réalité contemporaine de l'homosexualité est, en tant que telle, étrangère au domaine proche-oriental ancien ${ }^{66}$. Néanmoins, ces lectures présentent l'intérêt de revisiter d'une manière originale un passage biblique que nous lisons aujourd'hui sans y prêter beaucoup d'attention; elles amènent ainsi les critiques à préciser des aspects textuels sans doute pas assez explorés jusque-là. Elles ont encore le mérite de mettre en valeur des personnages qui pourraient passer quasiment inaperçus, même si elles leur donnent un rôle qu'ils n'ont pas joué. Tel est le cas d'Ashpenaz dans le livre de Daniel : cet « eunuque ", personnage secondaire s'il en est, n'est certainement pas le plus connu du livre prophétique. En étudiant ce personnage et son rapport à Daniel, nous avons été amenés à parcourir les exégètes modernes et surtout anciens : or, ce que montre une telle étude, c'est que les anciens s'étaient posé bien des questions semblables aux modernes; leurs réponses, cependant, diffèrent considérablement, et leur exposé en révèle toute la richesse et toute la profondeur. L'analyse du passage, loin de découvrir une relation homosexuelle entre Ashpenaz et Daniel, a permis au contraire de voir dans le chef des eunuques un fonctionnaire craignant le roi Nabuchodonosor, s'opposant à la volonté de Daniel et, par suite, à Dieu. C'est donc dans ce contexte d'opposition à la Loi divine que Dieu va pouvoir déployer, au travers de l'obstination des jeunes Hébreux, toute sa compassion et toute sa puissance.

$\mathrm{Au}$ moment même où nous achevions ces remarques paraissait un ouvrage sur l'homosexualité dans la Bible, traitant précisément encore de ce début du livre de Daniel, mais dans des perspectives quelque peu différentes. Cette coïncidence nous semble justifier tout à la fois les analyses qui précèdent et un examen des nouveaux arguments avancés par cet ouvrage ; nous souhaiterions donc, en épilogue, apporter quelques éléments d'analyse nouveaux.

\section{6. Épilogue : les surnoms des jeunes Hébreux}

\section{Discussions autour d'une explication récente}

En Daniel 1, 6 apparaissent pour la première fois quatre jeunes juifs dont les noms sont donnés : Daniel, Ananias, Misaël et Azarias ${ }^{67}$; dès le verset suivant, il est dit : « Le chef des eunuques leur imposa des noms: Daniel s'appellerait Baltassar, Ananias Shadrak, Misaël Meshak, et Azarias Abed Nego » (Dn 1, 7). C'est sur ce verset que Patrick Négrier s'arrête quelque peu longuement dans son ouvrage ${ }^{68}$. Sa thèse est que ces surnoms seraient des injures à connotation homosexuelle laissant supposer que les mœurs des quatre jeunes juifs - qui n'étaient en réalité pas forcément homosexuels - étaient dissolues; par ce stratagème, Ashpenaz - cette fois présenté comme un rival ${ }^{69}$ voudrait discréditer les jeunes enfants auprès du roi Nabuchodonosor. De fait, si le roi décide de faire instruire des jeunes juifs dans la langue et la littérature chaldéennes, c'est parce que les magiciens, devins et sorciers chaldéens s'étaient avérés incapables d'interpréter les songes du roi, en d'autres termes qu'ils étaient incapables de remplir correctement leurs fonctions administratives; Ashpenaz aurait donc peur de perdre 
son poste de chef des eunuques si les quatre enfants acquéraient une compétence supérieure à la sienne.

Cette thèse est d'autant plus séduisante que, comme le remarque l'auteur, le nom hébreu des jeunes enfants est lui-même porteur de sens: Daniel signifie en hébreu, selon Patrick Négrier, «La vertu est mon juge » (plus exactement ${ }^{70}$ : «Dieu est mon juge », « Dieu me juge » ou « Dieu a jugé »); Ananias, « l'Être éternel est grâce » («Dieu a fait grâce » ou « Dieu a eu pitié »); Misaël, « Qui est ce qu'est la vertu ?» (« Qui est ce que Dieu est? ? ou «Qui est ce que Dieu est »); Azarias, « l'Être éternel aide » («Dieu a aidé » ou « Dieu aide »). Dans chacun de ces noms apparaît le nom de Dieu sous la forme des suffixes -el (que l'on retrouve dans Élohim) ou -ias (plus exactement -iah, les deux premières lettres du tétragramme Yahvé). L'auteur lit dans ces quatre noms symboliques une «leçon spirituelle plurielle» que nous ne jugeons pas utile de rapporter ici. Se fondant en tout cas sur le fait que les noms d'origine des quatre jeunes gens ont une signification spirituelle, il se propose d'examiner les nouveaux noms qui leur sont attribués par Ashpenaz. Ces quatre surnoms auraient donc pour unique objet de discréditer les jeunes gens ${ }^{71}$ auprès du roi ; mais en fait, comme le note Patrick Négrier, c'est à chaque fois Ashpenaz qui manifeste son incapacité à occuper sa charge d'administrateur et d'éducateur.

Daniel est nommé Baltassar, ce qui signifierait en hébreu " Pas cul étroit ${ }^{72}$ ", " allusion à la pratique d'un coït anal passif répété »; l'injure concernerait moins le fait que Daniel pratiquerait l'homosexualité que le fait qu'il a des rapports sexuels anaux avec de nombreux partenaires. Peu importe l'argumentation biblique de l'auteur sur la condamnation par la Bible de la multiplication des rapports sexuels avec de nombreux partenaires $^{73}$, nous retiendrons seulement l'interprétation: «Lorsque le chef des eunuques donna à Danî'el le surnom infamant de "Pas cul étroit", il cherchait par là à attribuer à ce jeune homme la réputation d'un homosexuel qui, parce qu'il multiplie les rapports sexuels avec de nombreux partenaires, constitue de ce fait un agent de désordre social dommageable à la paix et à la sécurité publiques ${ }^{74}$. Intention qui permettrait au chef des eunuques de jeter le discrédit moral sur Danî'el, et par là de se débarrasser d'un jeune homme dont les compétences faisaient de lui un rival potentiel risquant de lui faire perdre sa place de chef des eunuques » (p. 214).

31 Ananias est nommé Shadrak, ce qui signifierait en hébreu «Poitrine douce ${ }^{75}$ ». Le chef des eunuques reprocherait ainsi à Ananias d'être comme une femme, soulignant ainsi son manque de virilité et donc sa féminité. L'auteur fait un long développement sur le rapport entre l'anatomie (qui définit le genre) et les traits psycho-moraux, que nous laissons de côté car il n'intéresse pas directement notre propos.

Misaël est nommé Meshak, ce qui signifierait en hébreu "Testicule écrasé ${ }^{76}$ ", «appellation connotant l'idée d'une virilité amoindrie et donc d'effémination». On peut relever deux allusions aux «testicules écrasées » dans la Bible : en Lévitique 21, 17-21, il est dit que tout descendant d'Aaron infirme ne pourra accéder au sacerdoce; parmi les infirmités citées, on trouve les testicules écrasées ( $\operatorname{Lv} 21,20)$. Toutefois, Meshak n'étant pas prêtre, ce verset ne pouvait lui faire perdre crédit auprès du roi. En revanche, Deutéronome 23, 2 («L'homme mutilé par écrasement et l'homme à la verge coupée n'entreront pas dans l'assemblée du Seigneur ») pouvait s'appliquer davantage à Meshak, bien que l'allusion à l'écrasement ne concerne pas explicitement les testicules. Ashpenaz cependant ferait une lecture superficielle de ce verset «pour révéler publiquement cet aspect de l'anatomie de ce jeune homme (conséquence 
éventuelle d'une rixe, et par là indice possible d'un naturel violent) en vue de le discréditer auprès du roi de Babylone, et écarter ainsi d'une carrière éminente ce jeune homme compétent qui représentait pour lui un rival suffisamment sérieux pour risquer de l'évincer de son propre poste de chef des eunuques ${ }^{77} »$ (p. 218).

Azarias est nommé Abed Nego, ce qui signifierait en hébreu "Esclave de son mignon ${ }^{78}$ ». Par ce nom, le chef des eunuques reprochait à Azarias « d'inverser les rôles dans sa relation avec son mignon, c'est-à-dire de tenir au plan psycho-moral un rôle passif de dominé alors que dans l'acte sexuel il tenait un rôle actif de dominant ». Ce reproche permettait «de le discréditer auprès du roi de Babylone en lui faisant accroire que 'Azariah n'était pas digne d'occuper au terme de ses études un poste de responsabilité administrative qui nécessite de tenir au plan psycho-moral un rôle actif de maître (c'est-à-dire de dominant) et non un rôle d'esclave (c'est-à-dire de dominé)» (p. 220-221). Suit un développement sur la racine 'azar- dans le nom hébreu du jeune homme qui signifie « aide » et que l'on retrouve en Genèse 2, 18 : son nom signifie ainsi "l'essence même de la relation de couple et de la relation entre administrateurs et administrés, comme le montra la suite du livre de Daniel en faisant état de la promotion professionnelle de "Azaryah au "service" ('avîdeta') de la province de Babel (Dan. 2, 48-49)» (p. 222).

L'argumentation proposée tombe, selon nous, dans deux écueils ${ }^{79}:$ d'une part, Patrick Négrier tente d'attribuer une étymologie hébraïque à des noms qui, de toute évidence, ne relèvent pas de cette langue (le verset 4 ne précise-t-il d'ailleurs pas : «Asphenaz leur enseignerait les lettres et la langue des Chaldéens"?). D'autre part surtout, l'ouvrage ne propose aucune référence bibliographique pour appuyer son raisonnement ${ }^{80}$; il passe ainsi sous silence les difficultés liées à l'étymologie de ces noms et les efforts faits par les exégètes, depuis au moins deux siècles, pour tenter de décrypter leurs significations; le lecteur prend donc pour argent comptant ce qui lui est exposé sans percevoir la complexité réelle posée par ces surnoms. Ces deux faiblesses méthodologiques nous conduisent à réexaminer les propositions de Patrick Négrier, si séduisantes soient-elles a priori ${ }^{81}$. Bien que l'auteur rejette, dans son introduction, les "ouvrages du passé qui avaient été conçus d'après des méthodes herméneutiques non pertinentes ${ }^{82}$ ", nous pensons cependant que l'on ne peut faire fi des travaux d'éminents chercheurs, même s'il convient toujours de les considérer avec esprit critique. C'est donc en nous appuyant sur ces travaux exégétiques que nous souhaiterions, aussi brièvement que possible, rétablir quelques positions ${ }^{83}$.

\section{Les explications apportées par les exégètes}

Pour les raisons que nous venons d'exposer, les exégètes n'ont pas cherché l'étymologie de ces noms en hébreu mais dans d'autres langues proche-orientales: l'une des idées qui a guidé ces recherches est que le chef des eunuques manifeste l'adoption des étrangers à la cour du roi de Babylone en leur attribuant des noms indigènes; or, puisque les noms des quatre enfants contenaient le nom de Dieu, les exégètes ont plutôt compris qu'Ashpenaz leur avait donné des noms contenant des éléments relatifs à la religion babylonienne, c'est-à-dire des noms idolâtres et profanes ${ }^{84}$; mais cette théorie n'est pas la seule et l'on a souvent avancé bien d'autres explications étymologiques. Nous n'avons aucune compétence pour exposer les diverses reconstitutions proposées ${ }^{85}$ 
et nous nous contenterons donc de les énumérer : le lecteur désireux d'approfondir cette question pourra se reporter aux travaux signalés dans les notes. d'exégètes ${ }^{100}$; et, même si l'on a tenté de découvrir son origine, celle-ci reste difficile à déterminer et très hypothétique. On a proposé d'y voir une altération intentionnelle d'un nom d'origine babylonienne, destinée à estomper un lien indésirable avec un dieu étranger ${ }^{101}$; il pourrait alors s'agir du nom cryptographique de Babel, Sheshaq - que l'on trouve en Jérémie 25,26 ou $51,41^{102}$; on a également pensé que le nom du dieu Marduk pouvait être contenu dans le $\operatorname{mot}^{103}$. Certains ont avancé une origine accadienne, associant le nom à la forme Mishaaku, «Qui est ce qu'est le dieu Aku ?» adaptation profane du nom Misaël ${ }^{104}$; pour d'autres, l'étymologie serait à rechercher du côté du vieux perse et serait un composé de miça, variante dialectale du nom Mithra ${ }^{105}$ explication rejetée par quelques-uns ${ }^{106}$. C'est encore vers l'accadien meshaku, «je suis méprisé107 » ou vers l'iranien *maisha, « mouton ${ }^{108}$ », que l'on s'est tourné, sans que ces explications parviennent à convaincre ${ }^{109}$. Enfin, on a vu dans ce surnom le nom d'un peuple localisé en Asie Mineure, dans la région du Pont, au sud de la mer Noire ${ }^{110}$ mais, là encore, l'explication n'a pas remporté les suffrages ${ }^{111}$.

Les quelques pages assez techniques qui précèdent n'avaient d'autre but que de montrer à la fois la grande complexité qui entoure l'origine de ces quatre noms (même si l'origine de deux d'entre eux semble recevoir le consensus des exégètes) et le vaste champ des investigations ouvert par la recherche. Au vu de cet éventail d'hypothèses aussi diverses qu'érudites, les explications proposées par Patrick Négrier doivent être 
remises à leur juste place: une hypothèse parmi d'autres. Le problème qu'elles soulèvent réside dans le fait qu'elles ne se présentent pas comme telles au lecteur. Ainsi, si l'objectif que se fixe l'auteur est louable - lutter contre l'homophobie, en l'occurrence les injures à connotation homosexuelle -, il apparaît que sa démonstration fondée sur Daniel 1, 7 ne repose pas sur des bases suffisamment solides pour emporter l'adhésion.

41 En guise de conclusion, on soulignera la place intéressante que peuvent tenir, dans l'examen des deux opinions discutées, les sources anciennes. Si l'exégèse contemporaine ne peut se satisfaire des seules opinions des commentateurs anciens, ceux-ci apportent ici un éclairage à la fois unanime et solide. Négliger ou nier la tradition, c'est risquer de dénaturer le texte biblique et d'avancer des opinions de manière certes séduisante, mais aventureuse. Or, il aurait été facile de se servir précisément de la tradition pour proposer, dans le même esprit, une autre figure « homosexuelle » dans le début de Daniel, en la personne du roi Nabuchodonosor luimême. De fait, dans ses Légendes des Juifs, Louis Ginzberg rapporte la tradition juive selon laquelle le roi s'adonnait «à des pratiques immorales au point qu'il fit souvent enivrer les rois prisonniers pour satisfaire sur eux ses passions contre nature ${ }^{112}$ ». Dans une note, l'auteur précise que le sens de ce passage est clair: Nabuchodonosor est accusé par le texte de s'adonner à la sodomie ${ }^{113}$. L'origine de cette légende est cependant claire : il s'agit de discréditer par avance le roi païen en le démarquant de ses prisonniers, lui qui « savait [...] que le Dieu des Juifs haïssait l'immoralité ${ }^{114}$ ».

\section{NOTES}

1. Voir R. COURTRAY (dir.), David et Jonathan. Histoire d'un mythe, coll. « Théologie historique » $\mathrm{n}^{\circ} 64$, Paris, Beauchesne, 2010..

2. Cf. D. HeLminiaK, Ce que la Bible dit vraiment de l'homosexualité, trad. par D. Gille, coll. "Les empêcheurs de penser en rond ", Paris, Le Seuil, 2005, p. 195-196 ( $1^{\text {re }}$ édition en anglais publiée en $2000)$; U. WERNIK, "Will the real homosexual in the Bible please stand up ?", Theology \& Sexuality 11/3, 2005, p. 47-64 (voir surtout les pages 56-57).

3. Non seulement cette nourriture ne répondait pas aux prescriptions de la loi mosaïque, mais les viandes risquaient d'avoir été consacrées aux divinités païennes.

4. Notre traduction littérale suit le texte proposé dans l'Ancien Testament interlinéaire hébreufrançais, Alliance biblique universelle, Villiers-le-Bel, 2007, p. 2424.

5. Cf. D. HELMINIAK, Ce que la Bible dit vraiment de l'homosexualité, p. 195.

6. Cf. U. WERNIK, "Will the real homosexual in the Bible please stand up ?", p. 56.

7. Une note précise cependant « matrices à la source de tout amour et de toute vie ».

8. Cf. J. MCNEILL, The Church and the Homosexual, Kansas City, Sheed Andrews and McMeel, 1976, p. 64-65.

9. Cf. N. WILSON, Our Tribe: Queer Folks, God, Jesus, and the Bible, San Francisco, Harper San Francisco, 1995, p. 124.

10. Cf. V.S. KOLAKOWSKI, "Throwing a party : patriarchy, gender, and the death of Jezebel”, in R.E. GOSs, et M. WEST (eds.), Take Back the Word, Cleveland, Pilgrim Press, 2000, p. 103. 
11. Cf. D. HELMiniaK, Ce que la Bible dit vraiment de l'homosexualité, p. 195.

12. Cf. U. WERNIK, "Will the real homosexual in the Bible please stand up ?", p. 56. Voir de même Si 20,4: «Tel l'eunuque qui voudrait déflorer une jeune fille » ou Jérôme, Lettres 107, 11, qui parle des eunuques qui «n'abandonnent pas leur âme de mâles ».

13. Pour les analyses qui suivent, nous avons eu recours à G. LISOWSKY, Konkordanz zum Hebräischen Alten Testament, Stuttgart, Deutsche Bibelgesellschaft, $1981^{2}$.

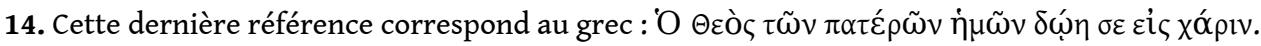

15. Cicéron, Tusculanes IV, $8,18$.

16. Ibid., IV, 7, 14.

17. André de Saint-Victor, Expositio super Danielem I, 9 (сссм 53F, éd. M. Zier, 1990, p. 11-12).

18. Cf. Jérôme, In Danielem 1, 1, 9 (CCSL 75A, p. 781, 1. 92-101).

19. Leçons de M. Jean Calvin sur le livre des prophéties de Daniel, recueillies fidelement par Jean Budé \& Charles de Jonviller, ses auditeurs et translatees de Latin en François, Genève, 1562, p. 7v-8r (sur Daniel 1, 9-10).

20. Sur les eunuques dans l'empire assyrien, voir, entre autres, A.K. GRAYSON, "Eunuchs in power : their role in the Assyrian bureaucracy", in M. DIETRICH et O. LORETZ (eds.), Vom alten Orient zum alten Testament, Alter Orient une Altes Testament 240, Neukirchen, 1995, p. 85-98 ; K. DELLER, “The Assyrian Eunuchs and their predecessors", in K. WATABE (éd.), Priests and officials in the Ancien Near East, Heidelberg, 1999, p. 303-311 ; F.M. FALES, L'impero assiro. Storia e amministrazione (IX-VII secolo A.C.), Roma, Laterza, 2001, passim.

21. Dictionnaire Encyclopédique de la Bible, Turnhout, Brepols, 2002, s.u. "eunuque”. D'autres dictionnaires voient une évolution inverse : «D’abord réservé aux serviteurs émasculés commis à la garde des harems chez tous les peuples antiques, puis dans les palais princiers ou royaux aux "gardes de la chambre", qu'ils aient ou non subi une mutilation, le terme finit par désigner tous les officiers qui tenaient une charge de confiance dans la maison du roi » (A.-M. GÉRARD, Dictionnaire de la Bible, Paris, Bouquins-Robert Laffont, 1989). Pour J.A. Montgomery (A Critical and Exegetical Commentary on the Book of Daniel, Edinburgh, T. \& T. Clark, 1927, p. 124), le fait que de hauts officiers militaires aient porté le titre d'eunuques ferait douter que le mot ait d'abord pu signifier "eunuque»; pourtant, il est plus facile de penser que le mot saris a évolué vers la signification d'un titre officiel que le contraire.

22. Cf. F.P. Retief, J.F.G. CILLIERS et S.P.J.K. RIEKERT, "Eunuchs in the Bible", Acta Theologica Supplementum 7, 2005, p. 247-258.

23. Certains lui prêtent même une fille, selon Genèse 41,45 ; mais il y a sans doute confusion entre le Putiphar, maître de Joseph, et un autre personnage, prêtre de Ône.

24. Voir de même en 2 R 18, 17 ; Jr 39, 3.13.

25. Leçons de M. Jean Calvin sur le livre des prophéties de Daniel, p. 3v (sur Daniel 1,3).

26. Ibid., p. 8 r (sur Daniel 1, 11-13). Cette explication est donnée à propos de Melzar (Amelsad), le garde préposé à Daniel et à ses compagnons.

27. D. HELIMINIAK, Ce que la Bible dit vraiment de l'homosexualité, p. 195.

28. Cf. Bruce L. GERIG, Eunuchs in the Old Testament, http ://epistle.us/hbarticles/eunuchs1.html/ (site consulté le 19 janvier 2011).

29. Cf. J. вотте́Ro et H. PEтsChow, "Homosexualité”, in Reallexikon der Assyrie, vol. IV, Berlin-Leipzig, Walter de Gruyter \& Co, 1972-1975, p. 459-468 (notamment les § 10-20, p. 463-468) ; J. воттÉRO, Mésopotamie. L'écriture, la raison et les dieux, Paris, Gallimard, 1987, p. 231-239.

30. J. Steinmann, Daniel, collection « Connaître la Bible », Paris, Desclée de Brouwer, 1961, p. 45.

31. Pour une étude plus approfondie des traditions juives sur cette question, voir J. BRAVERMAN, Jerome's Commentary on Daniel. A study of comparative Jewish and Christian interpretations of the Hebrew Bible, The Classical biblical Quarterly, Monograph Series 7, Washington 1978, p. 53-66 ; J. SMEETS, “Traditions juives dans la Vulgate de Daniel et le commentaire de Jérôme", Service international de 
Documentation judéo-chrétienne XII/2, 1979, p.21-22; L. GINZBERG, The Legends of the Jews, Philadelphia, The Jewish Publication of America, t. IV (1913), p. 276 ; 326 ; t. VI (1928), p. $368 ; 415$. 32. Is $39,7:$ : Parmi les fils issus de toi, ceux que tu as engendrés, on en prendra pour être eunuques dans le palais du roi de Babylone » (trad. Bible de Jérusalem).

33. Talmud de Jérusalem, Schabbath VI : Le Talmud de Jérusalem, trad. M. Schwab, Paris, éd. Maisonneuve et Larose, t. III, 1972, p. 80.

34. Midrash Megillah, dans Semitic Studies in Memory of Rev. Dr. Alexander Kohut, Berlin, 1897, p. 176 ; repris dans : J.D. Einsenstein, Otsar ha-Midrashim 1, New York, 1915, p. 606.

35. Le Midrah Megillah identifie l'eunuque Hatak d'Esther 4, 5 à Daniel, parce que hatak en hébreu signifie « trancher » et que Daniel et ses compagnons avaient tranché leur masculinité.

36. Flavius Josèphe, Antiquités Judaïques X, x, 1 (186-187), in Euvres complètes, trad. en français sous la dir. de Th. Reinach, trad. de René Harmand, révisée et annotée par S. Reinach et J. Weill, t. II, Paris, E. Leroux, 1926, p. 337-338.

37. Cf. A.-F. GALLÉ, Daniel, avec commentaires de R. Saadia, Aben-Ezra, Raschi, etc., et variantes des versions arabe et syriaque, Paris, E. Leroux, 1900, p. 4-5.

38. Cf. Origène, Fragmenta in librum primum Regnorum, frg. 22 (GCS 6).

39. Origène identifie ici le Daniel (ou Danel) mentionné en Ézéchiel 14,14 au prophète Daniel - ce que n'admettent pas les exégètes contemporains.

40. Cf. Origène, Selecta in Ezechielem (fragmenta e catenis) $(P G 13,808)$.

41. Cf. Origène, Homélie sur Ézéchiel IV, 5 et 8 (sc 352, p. $174 ; 184)$.

42. Cf. Théodoret de Cyr, Commentaire sur Daniel 1, 3-4 (PG 81, 1273C).

43. Cf. Épiphane, De uitis prophetarum 10, in T. SCHERMAN (éd.), Prophetarum uitae fabulosae, Leipzig, Teubner, 1907, p. 14, 1.8 ; p. 64, 1. 22.

44. Cf. Jérôme, Aduersus Iouinianum 25 (PL 25, 244B-245A). La question centrale de ce traité est celle de la virginité.

45. Le raisonnement de Jérôme repose sur une double erreur historique : d'une part - on l'a signalé à propos d'Origène -, le Daniel d'Ézéchiel 14 n'est pas le même que celui du livre de Daniel (il s'agit d'un juste de la tradition phénicienne que l'on trouve dans les textes mythologiques d'Ougarit - voir M. DELCOR, Le livre de Daniel, coll. "Sources bibliques », Gabalda, Paris, 1971, p. 63-64 ; A. LACOCQUE, Le livre de Daniel, Neuchâtel-Paris, Delachaux et Niestlé, 1976, p. 14-15 ; etc.) ; d'autre part, le moine confond le roi Joachim du livre de Daniel avec le roi Joiachin du livre d'Ézéchiel : il n'est donc pas possible de conclure que Daniel a été fait prisonnier en même temps qu'Ézéchiel. Or, en 407, dans son Commentaire sur Daniel, Jérôme - non sans une certaine mauvaise foi - mettra précisément en garde contre cette confusion inadmissible des deux rois, commise par Porphyre: «Personne ne saurait penser que ce Joachim que l'on trouve au début du livre de Daniel est le même homme que l'on rencontre au début du livre d'Ézéchiel écrit sous la forme Joiachin : le premier nom se termine par la syllabe "chim", le second par la syllabe "chin" » (In Danielem 1, 1, 1 - CCSL 75A, p. 777, 1. 11-14). Dans le Contre Jovinien, l'erreur de Jérôme semble provenir de l'utilisation du texte de la Septante qui confond les deux rois dans leur graphie. Pour une discussion plus détaillée de cette question, voir J. BRAVERMAN, Jerome's Commentary on Daniel, p. 63-64, n. 53.

46. Cf. Éz 8,$1 ; 14,1$ (Jérémie évoque Noé, Daniel et Job « la sixième année » de la déportation du roi Joachim). Tout ce raisonnement est sous-entendu par Jérôme qui procède par ellipse : au lecteur de compléter son argumentation.

47. Jérôme, In Danielem 1, 1, 3-4 (CCSL 75A, p. 779, 1. 51-59). Dans ses Quaestiones in Danielem prophetam (Quaestio 2, PL 96, 1348A), Pierre de Pise reprend, au VIII ${ }^{\mathrm{e}}$ siècle, l'argumentation de Jérôme, mais tranche une discussion que le moine avait laissée ouverte : on pourrait penser que Daniel et ses compagnons ont été eunuques, en rapprochant Daniel 1, 3 de Isaïe 39, 7, mais il faut conclure qu'ils n'ont pas été eunuques en raison de Daniel 1, 4 . 
48. Jérôme, In Danielem 2, 6, 4a (p. 830, 1. 260-p. 831, 1. 262).

49. Pour une discussion plus détaillée de ce passage, voir J. SMEETs, “Traditions juives...", p. 22-23. On trouve un parallèle intéressant à cette explication dans le Midrash Megillah.

50. Jérôme, In Isaiam XI, 39, 3-8 (cCSL 73, p. 453, 1. 50-54).

51. Dom Augustin CALMET, Commentaire littéral sur tous les livres de l'Ancien et du Nouveau Testament, t. 2 : Ézéchiel et Daniel, Paris, 1715, p. 560 (sur Daniel 1, 3).

52. B. BECQUET, F. DUMORTIER et M. JACOB, La fosse aux lions. Lecture du livre de Daniel, Paris, Les éditions ouvrières, 1993, p. 38.

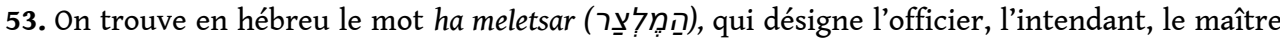
d'hôtel ou le surveillant (d'après N. Ph. SANDER et I. TRENEL, Dictionnaire hébreu-français, Genève, Slatkine Reprints, 1982). Toutefois, comme l'a bien noté M. Delcor (Le livre de Daniel, p. 65), « les versions anciennes ont été visiblement embarrassées en présence de ce terme. Théodotion a lu $A \mu \varepsilon \lambda \sigma \alpha \delta$ dont il fait apparemment un nom propre comme la LXX qui a lu A $\beta 1 \varepsilon \sigma \delta \rho l$. » On ne sera donc pas surpris, dans les textes qui suivront, que le nom propre Amelsad soit employé pour désigner ce garde. Voir sur les différentes interprétations J.A. MONTGOMERY, A Critical and Exegetical Commentary on the Book of Daniel, p. 131 et 134.

54. Cf. Théodoret de Cyr, Commentarius in uisiones Danielis prophetae 1, 10 (PG 81, 1277B-C).

55. Cf. Jean Chrysostome, Interpretatio in Danielem prophetam $1,9(P G 56,197)$.

56. Cf. Éphrem, Sermo in Danielem prophetam, et in sanctos tres pueros; et in eum qui dicit: 'Tempora

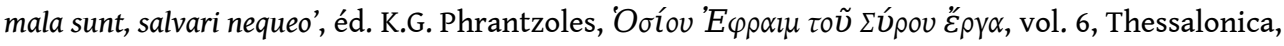
To Perivoli tis Panagias, 1995 (p. 65-69), 1. 28-35.

57. Cf. Sulpice Sévère, Chroniques II, I, 1 ( $s c 441$, p. 222, 1. 16).

58. Littéralement : « La foi assista ces paroles. »

59. Toutefois, le genre même des Chroniques et la concision que ce genre implique ont amené Sulpice Sévère à simplifier les épisodes, éliminant au passage les personnages secondaires, comme celui du garde.

60. Flavius Josèphe, Antiquités Judaïques X, X, 2 (191-193), p. 338-339.

61. Selon l'orthographe donnée par Flavius Josèphe.

62. Leçons de M. Jean Calvin sur le livre des prophéties de Daniel, p. 7r (sur Daniel 1, 9-10).

63. Dom Augustin CALMET, Commentaire littéral..., t. 2, p. 565 (sur Daniel 1, 11).

64. Voir notamment, sur le changement de nom : Jérôme, In Danielem 1, 1, 7 (cCSL 75A, p. 780, 1. 70-71); sur la bienveillance du geôlier: Théodoret de Cyr, Commentarius in uisiones Danielis prophetae 1,9 (PG 81, 1277A).

65. J. STEINMANN, Daniel, p. 43-45.

66. Voir, par exemple, T. RÖMER et L. BONJOUR, L'homosexualité dans le Proche-Orient ancien et la Bible, Genève, Labor et Fides, 2005 ; I. HIMBAZA, A. SCHENKER et J.-B. EDART, Clarifications sur l'homosexualité dans la Bible, Paris, Cerf, 2007.

67. Nous avons adopté ici l'orthographe des noms établie par la Bible de Jérusalem; bien des variantes existent selon les Bibles, selon que l'on tente de donner une graphie plus proche de l'hébreu ou du français.

68. P. NÉGRIER, Contre l'homophobie. L'homosexualité dans la Bible, Paris, éditions Cartouche, 2010, p. 205-228.

69. Son nom signifierait, d'après l'auteur: «Carquois de flèches qui éclaboussent. " Sans le

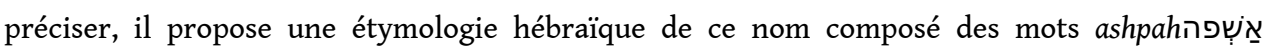
(« carquois ») et nazahLes exégètes rapprochent plutôt. (« rejaillir »; au hiphil : « asperger ») ce nom du mot perse Ashpazanda dont le sens reste difficile à expliquer (cf. E.J. YounG, The Prophecy of Daniel. A Commentary, Grand Rapids, W.M.B. Eerdman, $1962^{2}$, p. 39 ; A. LACocQUE, Le livre de Daniel, p. 30 ; J. SteInMAnN, Daniel, p. 41 ; etc.). 
70. Nous rectifions entre parenthèses les traductions au plus près de l'étymologie hébraïque, en nous appuyant sur les ouvrages suivants: B. DAviDson, The Analytical Hebrew and Chaldee Lexicon, London, 1848, réimpr. Grand Rapids, Zondervan, $1993^{2}$; F. VIGouRoux, Dictionnaire de la Bible, 5 vol., Paris, Letouzay et Ané, 1895-1912 ; J.A. MONTGOMERY, A Critical and Exegetical Commentary on the Book of Daniel ; O. ODELAIN et R. SÉGUINEAU, Dictionnaire des noms propres de la Bible, Paris, Le Cerf-Desclée de Brouwer, 1978; N.Ph. SANDER et I. TRENEL, Dictionnaire hébreu-français, Genève, Slatkine Reprints, 1982 ; D.N. FREEDMAN (éd.), The Anchor Bible Dictionary, 6 vol., New York-London-TorontoSydney-Auckland, Doubleday, 1992 ; Dictionnaire Encyclopédique de la Bible, Turnhout, Brepols, 2002.

71. Ceux-ci sont donc supposés être assez âgés pour avoir des relations sexuelles - ce que le texte biblique ne permet pas de confirmer, parlant de

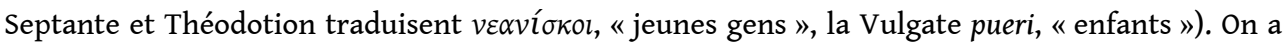
vu plus haut les arguments (erronés) de Jérôme; André Lacocque (p. 30) indique que le terme s'applique aussi à des adolescents (par exemple : Gn 37, 30 - à propos de Joseph !) et rapporte que, pour Ibn Ezra, Daniel avait alors plus ou moins 15 ans.

72. Cette analyse étymologique repose sur des racines hébraïques, mais aussi sur la modification de l'une des parties du mot: bel-tesha'-tsar serait à lire plutôt sous la forme bel-sht-tsar, bal(בבָּ) signifiant «ne pas », shet anus » et » (שֵֶ) tsarétroit »; la modification proposée est due au ») fait que la forme tescha ne signifie rien en hébreu (on pourrait cependant proposer la traduction : "tu porteras", selon le dictionnaire de Davidson: תִשאט). P. Négrier découvre une preuve supplémentaire en 1 Chroniques 4,12 où il lit le mot Éshtôn (אֶֶֶון) qui aurait pour sens «homme efféminé » (racine אֶֶ, «femme »); or, il s'agit d'un nom propre, dont l'étymologie viendrait plutôt de אִ̣ (« homme»).

73. Cette condamnation serait fondée sur Genèse 6, 1-4 et concernerait avant tout les rapports hétérosexuels. Le passage incriminé ne nous semble pas probant.

74. Cette idée repose toujours sur Genèse 6 : ce serait en raison d'une multiplication de relations sexuelles avec de nombreux partenaires que le déluge se serait produit; par déluge, il faut comprendre une guerre civile « qui faillit exterminer l'existence de l'espèce humaine » selon la métaphore mésopotamienne du déluge pour signifier les ravages opérés par la guerre. Il en irait de même pour Daniel.

75. Le nom serait ainsi composé de shad,non pas « poitrine », mais « sein, mamelle » (ce qui , שِ (שָד) du coup, ne convient plus à l'analyse qui est faite du mot) et de rak.« tendre, mou, délicat ", (עד) (עד) (עד) L'auteur cite Deutéronome 28, 54 où le mot rak apparaît, associé au terme 'anogque l'on (עַjג) trouverait précisément dans le nom Abed Nego. Rien cependant n'autorise a priori à affirmer que l'expression «l'homme tendre» signifie "l'homme sexuellement tendre»: de fait, non seulement l'homme en question possède femme et enfants, mais en Deutéronome 28, 56, la même structure de phrase est appliquée à une femme (l'auteur a complètement passé sous silence le contexte, selon lequel il est dit que les plus doux - hommes et femmes - deviendront les plus féroces, si le peuple n'écoute pas la voix du Seigneur). L'argument sur lequel se fonde P. Négrier est l'équivalence entre le terme rak et le mot grec malakos. Laissons de côté les développements sur le terme grec qui nous entraîneraient trop loin de notre objet; contentons-nous de considérer l'affirmation selon laquelle «le modèle typologique de la malakia néotestamentaire était le qualificatif rak ("doux") employé en Dan. 1, 7 et en Deut. 28, 54 dans un contexte d'homosexualité ». Pour qu'une telle affirmation puisse être exacte, il faudrait démontrer que les emplois néotestamentaires des mots malakos ou malakia prennent effectivement appui sur ces deux passages ; or, c'est impossible à montrer pour Daniel (puisque la racine est insérée dans un nom qui sera transcrit directement en grec) et, pour ce qui est du Deutéronome, le mot "doux » est traduit par hapalos ( $\left.\dot{\alpha} \pi \alpha \lambda \hat{\sigma}_{\varsigma}\right)$ dans la Septante : l'absence de correspondance des termes suffit à notre sens pour refuser l'assimilation des deux mots et les développements qui en sont tirés. 
76. Le nom serait la forme abrégée de merôah et de 'ashek; les deux mots se trouvent de fait employés en Deutéronome 21, 20 : אֶׁשֶך (" homme aux testicules écrasées »). Si l'expression est bien attestée, on peut toutefois s'étonner d'une telle forme abrégée et se demander comment des lecteurs de la Bible auraient pu reconnaître dans la seule syllabe me- la racine du verbe signifiant « écraser ».

77. On comprend cependant mal en quoi Nabuchodonosor aurait pu se sentir concerné par un interdit lié aux assemblées cultuelles.

78. Le nom serait composé du mot 'abed signifiant " esclave ", "serviteur » et du mot (עִבְבד (anog (), «tendre, délicat» sous la forme 'onegô. Outre le fait que les voyelles ne correspondent à aucune forme attestée, il faudrait encore expliquer comment le 'ayin initial disparait.

79. Nous ne commenterons pas ici la conclusion ("Perspective typologique») dans laquelle P. Négrier considère que la corne de la bête dont Daniel a la vision (Dn 7, 8 - ce verset est rapproché d'Ap 13,5) est à rapporter à Ashpenaz : la bouche placée sur la corne et disant des énormités serait une allusion aux éclaboussures du chef des eunuques sur les quatre juifs. Le passage est cependant clairement apocalyptique et ne saurait faire une allusion à un épisode aussi peu significatif que Daniel 1, 7.

80. L'auteur s'explique sur l'absence de bibliographie dans son introduction (p.35-36), son ouvrage constituant « une révolution herméneutique qui appelle, de ses vœux, [...] l'élaboration de nouvelles interprétations de la Bible désormais prêtrs à utiliser à leur tour la méthode herméneutique dont nous fournissons un exemple appliqué dans le présent essai » (p. 36).

81. La séduction de ces étymologies tient à la cohérence des significations proposées pour chacun des quatre noms.

82. P. NÉGRIER., p. 36.

83. Outre les travaux mentionnés à la note 70 , nous avons eu recours aux notes des traductions modernes de la Bible (Bible de Jérusalem, TOB, Osty, Pléiade, Chouraqui) ; à G.A. BUTTRICK (éd.), Interpreter's Dictionary of the Bible, 4 vol., Nashville, 1962 ; aux ouvrages de M. DELCOR, Le livre de Daniel; A. LAcocoue, Le livre de Daniel; J. Steinmann, Daniel, de H. Golwurm, Daniel. Traduction et commentaires fondés sur les sources talmudiques, midrachiques et rabbiniques (trad. J.-J. Gugenheim), éd. du Sceptre, Paris 2001; aux articles de P.-R. BERGER, "Der Kyros-Zylinder mit dem Zusatzfragment BIN II Nr. 32 und die akkadischen Personennamen im Danielbuch", Zeitschrift für Assyriologie 64, 1975, p. 192-234 (voir p. 226-234) et de R. ZADOK, "Short Notes on five iranian names in the Old Testament", Vetus Testamentum 26, 1976, p. 246-247; au compte-rendu d'É. LIPIN SKI, “André Lacocque, Le Livre de Daniel", Vetus Testamentum 28, 1978, p.234-235. Cette bibliographie ne se prétend bien entendu pas exhaustive. Pour les dictionnaires, le lecteur se rapportera directement aux articles concernant les noms examinés ; pour les notes des Bibles, il se reportera à Daniel 1,7 .

84. Cf. J.A. MONTGOMERY, A Critical and Exegetical Commentary on the Book of Daniel, p.123; H. GoldwuRm, Daniel, p. 65. Les Pères de l'Église ont fait cette même remarque : cf. Théodoret de Cyr, Commentaire sur Daniel 1, 7 : «Il leur donna des noms chaldéens, puisque le roi avait également ordonné que les jeunes gens apprennent la langue chaldéenne» $(P G 81,1276 \mathrm{~B})$; Jérôme, In Danielem 1, 1, $7:$ : Le chef ou maître des eunuques, et, selon d'autres traducteurs, l'archieunouchos fait changer de nom aux saints, mais il n'est pas le seul : Pharaon aussi a appelé Joseph, quand il était en Égypte, Çophnat-Panéah (Gn 41,45); c'est que tous deux voulaient qu'en terre de captivité, ils ne portent pas un nom juif»; à ces noms pervertis, le moine oppose des changements de noms « dans un sens favorable » opérés par Dieu : «Quant au Seigneur, il change les noms anciens dans un sens favorable et d'après les circonstances, il donne des noms de vertus, appelant Abram Abraham, Saraï Sara (Gn 17, 5.15); dans l'Évangile aussi, à un certain moment, Simon a reçu le nom de Pierre (Mt 10, 2;16, 17-18; Mc 3, 16 ; Lc 6, 14 ; Jn 1, 42), et les fils de Zébédée sont appelés “fils du tonnerre", parce qu'on ne lit pas, comme le pensent la 
plupart des gens, “boanerges”, mais, plus correctement "banereem” (Mc 3, 17) » (cCSL 75A, p. 780, 1. 68-79).

85. Nous citerons les reconstitutions proposées en simplifiant au maximum les graphies (il se peut que nous commettions des inexactitudes de transcription: celles-ci n'auront toutefois aucune conséquence pour notre exposé).

86. Voir, entre autres: J.A. MONTGOMERY, A Critical and Exegetical Commentary on the Book of Daniel, p. 129 ; A. LACOCQUE, le livre de Daniel, p. 35 ; o. oDELAIN et R. SÉGUINEAU ; Dictionnaire Encyclopédique de la Bible; notes de la тов, de la Pléiade.

87. Cf. J.A. MONTGOMERY, A Critical and Exegetical Commentary on the Book of Daniel, p. 123, 129; The Anchor Bible Dictionary; note de Chouraqui.

88. Cf. J. SteInMANn, Daniel, p. 41 ; M. DelCoR, Le livre de Daniel, p. 64 ; A. LACOCQUE, le livre de Daniel, p. 35 ; J.A. MONTGOMERY, A Critical and Exegetical Commentary on the Book of Daniel, p. 129.

89. Cf. Dictionnaire de la Bible; The Anchor Bible Dictionary; J.A. MONTGOMERY, A Critical and Exegetical Commentary on the Book of Daniel, p.129. En revanche, le nom du roi Balthazar viendrait de l'accadien Bel-shar-usur et signifierait : « Bel, protège le roi ! » (O. ODELAIN et R. SÉGUINEAU).

90. Cf. J.A. MONTGOMery, A Critical and Exegetical Commentary on the Book of Daniel, p. 129.

91. Cf. P.-R. BERGER, "Der Kyros-Zylinder".

92. Tous les travaux consultés sont d'accord sur ce point. Seul P.-R. Berger rattacherait ce nom à la racine $n q w$ du sémitique oriental signifiant « luire ».

93. Cf. note d'Osty.

94. Cf. M. DelCor, Le livre de Daniel, p. 64.

95. Cf. J. STEINMANn, DANIEL, p. 41 ; The Anchor Bible Dictionary; J.A. MONTGOMERY, A Critical and Exegetical Commentary on the Book of Daniel, p. 123, 129 (ce dernier rapproche le nom de Daniel 1, 7 du nom du dieu appelé Nisrok en 2 Rois 19, 37, qui désignerait sans doute aussi le dieu Marduk).

96. Cf. Dictionnaire de la Bible; J.A. MONTGOMERY, A Critical and Exegetical Commentary on the Book of Daniel, p. 129.

97. Cf. note de Chouraqui.

98. Cf. R. ZADOK, “Short Notes...”, p. 247 ; É. LIPIŃSKI, “André Lacocque, Le Livre de Daniel”, p. 314 ; Dictionnaire Encyclopédique de la Bible.

99. Cf. P.-R. BERGER, "Der Kyros-Zylinder".

100. Cf. J.A. MONTGomery, A Critical and Exegetical Commentary on the Book of Daniel, p. 123 ; notes d'Osty, de Chouraqui.

101. Cf. J.A. MONTGOMERY, A Critical and Exegetical Commentary on the Book of Daniel, p. 129 ; The Anchor Bible Dictionary.

102. Cf. J.A. MONTGomery, A Critical and Exegetical Commentary on the Book of Daniel, p. 129; A. LACOCQUE, Le livre de Daniel, p. 35 ; J. STEINMANN, Daniel, p. 41.

103. Cf. J.A. MONTGOMERY, A Critical and Exegetical Commentary on the Book of Daniel, p. 129.

104. Cf. The Anchor Dictionary ; Interpreter's Dictionary on the Bible; M. DELCOR, Le livre de Daniel, p. 64.

105. Cf. É. LIPIŃSKI, p. 234 ; Dictionnaire Encyclopédique de la Bible.

106. Cf. Dictionnaire de la Bible.

107. Cf. P.-R. BERGER, "Der Kyros-Zylinder".

108. Cf. R. ZADOK, “Short Notes...", p. 246.

109. Cf. É. LIPIŃSKI, “André Lacocque, Le Livre de Daniel”, p. 234.

110. Cf. A. LACOCQUE, Le livre de Daniel, p. 35 (s'appuyant sur Hérodote III, 94 ; VII, 78) ; note de la тов.

111. Cf. É. LIPIŃSKI, “André Lacocque, Le Livre de Daniel”, p. 234.

112. L. GINZBERG, Les légendes des Juifs, t. VI, Paris, Le Cerf-Institut Alain de Rothschild, 2006, trad. G. Sed-Rajna, p. 116. 
113. Ibid., p. 301, n. 107. Ailleurs (p. 237, n. 16), l'auteur cite des midrashim montrant encore que, si Nabuchodonosor, Pharaon, Hiram et Joas revendiquèrent le fait d'être des dieux, ils finirent par être traités comme des femmes (coitu faeminico commixantur).

114. Ibid., p. 116.

\section{RÉSUMÉS}

Le premier chapitre du livre de Daniel a été l'objet - comme d'autres passages de la Bible - de lectures nouvelles au cours de ces dernières années. Certains ont vu dans la relation entre l'eunuque Ashpenaz et Daniel déporté à la cour de Babylone, une relation homosexuelle; d'autres ont vu dans le surnom donné aux trois jeunes gens des noms à caractère homophobe. Il s'agit là de lectures engagées, qui cherchent à démontrer que le texte biblique porte un regard bienveillant sur l'homosexualité et condamne l'homophobie. Reprenant l'ensemble du dossier, le présent article analyse les versets bibliques utilisés, s'appuyant sur les sources anciennes et des études contemporaines pour apporter un éclairage scientifique et historique, et décider si de telles lectures sont fondées et possibles.

The first chapter of the book of Daniel has been the object -like other passages in the Bible- of new readings in the course of recent years. Some have seen in the relationship between the eunuch Ashpenaz and Daniel deported to the court of Babylon a homosexual relationship; others have read in the nicknames given to the three young men names of a homophobic nature. Those are committed readings which seek to demonstrate that the biblical text casts a benevolent eye on homosexuality and condemns homophobia. Going over the whole dossier, the present article analyzes the biblical verses referred to and relies on ancient sources and contemporary studies in view of bringing a scientific and historical light and deciding whether such readings are founded and possible.

\section{INDEX}

Mots-clés : Livre de Daniel, exégèse contemporaine, Ashpenaz, eunuque, homosexualité, homophobie

Keywords : Book of Daniel, contemporary exegesis, Ashpenaz, eunuch, homosexuality, homophobia

\section{AUTEUR}

\section{RÉGIS COURTRAY}

Université de Toulouse (UTM)

PLH-CRATA

courtray@univ-tlse2.fr 